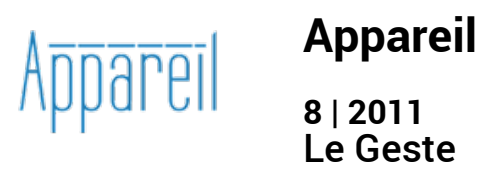

\title{
Quand Foucault dit « nous »...
}

Alain Brossat

\section{OpenEdition}

Journals

Édition électronique

URL : http://journals.openedition.org/appareil/1265

DOI : 10.4000/appareil. 1265

ISSN : 2101-0714

Éditeur

MSH Paris Nord

\section{Référence électronique}

Alain Brossat, «Quand Foucault dit « nous »... », Appareil [En ligne], 8 | 2011, mis en ligne le 26 octobre 2011, consulté le 30 juillet 2020. URL : http://journals.openedition.org/appareil/1265 ; DOI : https:// doi.org/10.4000/appareil.1265

Ce document a été généré automatiquement le 30 juillet 2020.

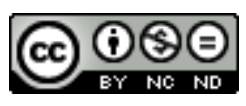

Appareil est mis à disposition selon les termes de la Licence Creative Commons Attribution - Pas d'Utilisation Commerciale - Pas de Modification 4.0 International. 


\title{
Quand Foucault dit « nous »...
}

\author{
Alain Brossat
}

1 Cet exposé est né d'un certain agacement, et même d'un agacement certain, suscité par l'assurance avec laquelle un certain nombre de critiques contemporains de Foucault, situés dans le champ des études post-coloniales, considèrent comme indiscutable la qualification de sa position philosophique comme "eurocentrique ", un terme auquel on ajoutera parfois l'adjectif " extrême " pour faire bonne mesure, "occidentaliste ", etc. La manière dont, dans certains textes du moins, ce procès est instruit me paraît si expéditive et si mal argumentée que j'ai décidé de revenir sur ce qui constitue quelque chose comme un «choix originel» (la formule est de Foucault lui-même) ou un geste premier établi au fondement de son travail - une certaine façon d'en délimiter l'espace, plutôt que le territoire, et de lier à cette approche topologique un art d'énoncer un « nous » tout à fait singulier.

2 Le geste premier de Foucault, dans ses grands livres-enquêtes soutenus par le fil conducteur de l'archéologie, Histoire de la folie à l'âge classique, Les mots et les choses, La volonté de savoir (et d'autres) consiste en un rejet décidé de ce qui constitue l'élément moteur ou le pli du discours philosophique dans sa tradition la plus immémoriale: l'établissement de celui-ci dans un horizon où les énoncés sont supposés valoir en général, quel que soit l'objet dont il est question - l'homme, la morale, la culture, la raison ou la folie, etc. Lorsque Foucault dit et répète à l'envi qu'il «n'est pas philosophe ", c'est bien ainsi qu'il faut entendre ce propos: on ne peut faire de la philosophie de cette façon, sur ce mode, sous cette condition de généralité ou d'universalité supposée de tous les énoncés proposés. Le sujet transcendantal doit être reconduit à ses conditions de relativité à une historicité, une temporalité, une spatialisation qui définissent des conditions de possibilité, en termes d'économie du discours, de partage entre le vrai et le faux... Si l'on peut encore faire de la philosophie ou quelque chose qui, d'une manière ou d'une autre, en proroge le geste, alors ce ne peut être qu'à la condition d'une auto-destitution (plutôt que décentrement) consistant pour l'énonciateur philosophique à expliciter les découpes que supposent ses descriptions et affirmations et à faire revenir sans cesse dans le jeu l'élément du relatif. 
3 En d'autres termes, le jeu de «dédisciplinarisation » de la philosophie (de destitution de la philosophie universitaire) auquel s'adonne Foucault va consister à se déplacer du côté d'autres disciplines ou domaines de savoirs et à adopter certains de leurs gestes premiers: la géographie (toute recherche philosophique doit être spatialisée, cartographiée), l'ethnographie (l'élément de la culture humaine est la pluralité, la relativité d'un ensemble à tous les autres), l'histoire (la vérité a une histoire), etc. En cessant de devenir l'administrateur des vérités générales, le philosophe se rapproche paradoxalement de cela même dont il s'est fait l'archéologue - les sciences humaines (le sous-titre de Les mots et les choses) : il devient, fondamentalement un enquêteur, comme le sont l'historien, le géographe, l'ethnographe ou l'anthropologue bien sûr, etc. Et qui dit enquêteur signale bien ce nouveau pacte entre une recherche (un geste) qui, envers et contre tout, s'origine bien dans la tradition philosophique et l'élément du singulier, du spécifique, du discontinu - bref tout ce qui présuppose le multiple plutôt que l'un et s'impose comme condition ce que j'appelais les découpes - le choix d'un objet qui suppose l'exclusion de tous les autres, mais ceux-ci n'étant pas ignorés ou rejetés, mais au contraire identifiés comme l'extérieur légitime du champ de la recherche. Découpes : Histoire de la folie à l'âge classique - la deuxième partie du titre présente ce que j'appelle une découpe, Les mots et les choses - une archéologie des sciences humaines - autre découpe, pas les sciences en général, etc.

Ce parti adopté, il faut nommer les espaces découpés, c'est-à-dire topographier l'objet de la recherche, en baliser le champ - un travail de géomètre. L'objet se forme au point de rencontre de l'axe diachronique et de l'axe synchronique, par la combinaison de trois termes. Soit, si l'on prend la première grande "enquête" de Foucault - Folie, âge classique, culture européenne - un agencement dont le titre de l'ouvrage ne rend compte que de façon imparfaite - Histoire de la folie à l'âge classique - le terme " histoire " étant susceptible de susciter ici toutes sortes de malentendus. Mais, étrangement, ce n'est pas sur ce point que l'opération de découpe pratiquée par Foucault apparait litigieuse aux yeux de ceux qui soutiennent l'incrimination d'« eurocentrisme » ou " occidentalisme ». Ce qu'ils contestent avec véhémence, c'est l'emploi sans précaution par Foucault de termes ou expressions comme «l'Occident ", «le monde occidental ", « la culture occidentale ", «l'Europe », « la culture européenne », « notre civilisation », « notre modernité », « les sociétés modernes occidentales », (etc.) qui, toutes, sous leurs apparences purement descriptives, comporteraient tout un impensé pernicieux déterminé par les conditions mêmes (historiques, culturelles, politiques...) dans lesquelles ces notions s'imposent comme fallacieusement naturelles. Pour l'essentiel, ce qui est en cause, c'est l'impensé de la colonisation dans la constitution même des systèmes de représentation fondés sur le couplage et l'opposition entre des entités supposées compactes comme l'Occident et l'Orient, L'Europe et ce qui s'en séparerait ou s'y opposerait...

5 Ce qui est incriminé, c'est la supposée naïveté culturaliste de Foucault qui, tout promoteur qu'il est du projet archéologique (et de la disposition généalogique qui ne s'en sépare guère) appliqué aux sciences humaines, se garderait bien de faire tomber sous le coup du règlement archéologique des notions qui comptent parmi les « nombres premiers » de toutes ses enquêtes (la constance de leur emploi traverse les différentes topiques de l'œuvre de Foucault).

6 Cette objection serait forte si elle visait à démontrer que cette sorte de désinvolture conceptuelle avec laquelle Foucault recourt à ces notions pesait sur les résultats de ses 
recherches, les pré-déterminait, en quelque sorte. Mais ce n'est évidemment pas le cas, la contamination de l'analyse du Grand Renfermement ou bien celle des conditions dans lesquelles les mots s'entrecroisent avec les représentations, dans l'epistémè de l'âge classique par l'absence de généalogie d'une notion comme celle de «culture européenne » telle que l'emploie Foucault, sur un mode purement topologique, n'étant pas chose facile. Mais ce n'est pas ce qui, en l'occurrence, intéresse ceux qui opposent à l'ensemble du travail de Foucault cette sorte d'objection préalable. Ce qui leur importe est beaucoup plus de faire valoir de nouvelles conditions, de nouvelles normes, un nouveau règlement discursif et dont ils seraient les gardiens légitimes: une normativité dont le fondement est le soupçon : tout l'édifice de la recherche de Foucault étant établi sur des fondations aussi suspectes que les notions incriminées, il n'est pas même nécessaire d'entrer dans des démonstrations circonstanciées pour en dénoncer le travers "eurocentrique ", "occidentaliste ». Plus expéditivement encore, on ira jusqu'à asséner cet «argument »: la preuve irrécusable de ce travers constitutif de l'œuvre de Foucault, c'est que tous ses terrains, toutes ses enquêtes, tous ses objets de recherche sont situés dans ce pré carré «européen» ou "occidental», ce qui manifesterait crûment son incapacité à affronter la question de l'« hybride » ou, tout simplement, celle de l'altérité.

Difficile d'imaginer approche plus biaisée du travail de Foucault. Ce qui caractérise en premier lieu le dispositif d'enquête et de production de la connaissance mis en place par Foucault, c'est la destitution préventive de toute présomption de centralité, implicite ou explicite de ce qu'il découpe comme son objet de recherche - que cette présomption porte les habits de « la capitale » (Derrida), de l'universel (Castoriadis) ou du souverain. Foucault accorde donc à des termes ou expressions comme "l'Europe ", « la culture européenne » le même statut strictement descriptif et nullement normatif que lorsque Lévi-Strauss auquel il se réfère souvent lorsqu'il aborde ces questions, dit «Les Bororos » ou Pierre Clastres « Les Guayakis ». Le fait même de poser l'opération de la découpe comme condition a priori de l'enquête (du geste philosophique redéployé) est inséparable de l'opération de destitution du préjugé de centralité. Lorsque Foucault écrit, dans Histoire de la folie..., «Il a été sans doute essentiel à la culture occidentale [je souligne, AB] de lier comme elle l'a fait sa perception de la folie aux formes imaginaires du rapport de l'homme à l'animal ", il veut bien dire : ici et pas ailleurs, il explicite bien la restriction selon laquelle ses énoncés sont soumis aux conditions d'une géophilosophie, d'une philosophie qui s'est approprié le geste du géomètre. Lorsque dans Les mots et les choses, il parle de l'« epistémè moderne comme sol positif de notre [je souligne] savoir ", c'est le même type de condition rigoureuse de délimitation qu'il énonce, s'imposant à tout énoncé et en balisant le champ de validité. Lorsque, dans $L a$ volonté de savoir, il soutient que «l'aveu est devenu en Occident [je souligne, AB] une des techniques les plus hautement valorisées pour produire le vrai ", il fait davantage que suggérer qu'il existe d'autres configurations sociales et culturelles dans lesquelles d'autres techniques de production du vrai imposent leurs conditions - la discontinuité, la pluralité et l'hétérogénéité étant ici des notions qui s'imposent comme antidote au persistant préjugé de centralité. Pour dire les choses simplement, Foucault opère dans l'espace de la philosophie le même type de révolution que Lévi-Strauss dans celui de l'anthropologie ou l'ethnographie, affirmant après Boas le statut de belle totalité de toute culture, irréductible à toute autre, relative à toute autre, une parmi les autres, irréductiblement « dé-centrée " à ce titre. Ce que ne comprennent pas ceux qui font à Foucault ce mauvais procès en "occidentalocentrisme », c'est qu'il n'y a pas moins de 
tranchant, en philosophie, dans le geste consistant à dévoiler les conditions culturelles implicites qui fondent la prétention du sujet tout court de la connaissance à dire le vrai (c'est le sens du célèbre passage sur Descartes et la folie dans Histoire de la folie) qu'il y en a, en anthropologie, à renoncer à la notion même d'une hiérarchie des cultures et des formes d'organisations sociales. C'est une sorte de contresens absolu sur le geste même inauguré, en philosophie, par Foucault, que celui qui détecte dans le trait endogène de ses recherches (un trait qui fonde le va-et-vient entre la géographie objective et la géographie subjective - "l'Occident», «l'Europe» d'une part, «nos sociétés », notre culture », "nous » de l'autre) l'indice de la persistance du préjugé de centralité. C'est l'exact opposé qui est vrai : qu'il travaille sur l'hôpital général, le statut du langage à l'âge classique ou la scientia sexualis, ce que Foucault vise à exposer est bien une singularité, toujours susceptible de devenir une bizarrerie aux yeux des autres, comme l'est aux nôtres la fameuse « encyclopédie chinoise » de Borges. Il n'est pas un des livres de Foucault parmi ceux que j'ai mentionnés jusqu'ici qui ne constitue une invitation qui nous (Français, européens, occidentaux...) amène à re-considérer tout le domaine du familier en le passant au prisme de l'« encyclopédie chinoise » de Borges. Ce n'est pas pour rien que Foucault travaille pendant la majeure partie de sa carrière de chercheur sur des objets litigieux, sombres - comme la folie, la prison, la maladie, la sexualité, objets à propos desquels il fait émerger des spécificités du procès de civilisation dans lequel il s'inclut, objets dont il s'acharne à mettre en relief le trait d' inquiétante étrangeté, en les soumettant à la méthode archéologique notamment - ceci plutôt que de tenter de faire de l'Europe ou de l'Occident le sol natal de notions et "valeurs » destinées tout naturellement à conquérir le monde en faisant valoir leurs titres à l'universalité - le citoyen, la démocratie, les droits de l'homme, etc. - c'est la posture la plus courante de la philosophie politique et des sciences politiques réunies.

8 Lévi-Strauss apostrophe le public occidental - il va falloir vous faire à l'idée qu'il n'y a pas moins de "génie » humain dans le système de parenté, les récits et le mode de vie de la plus humble tribu amazonienne que dans la plus "avancée " des sociétés industrielles; Foucault inverse l'interpellation: parcourons ensemble les caves, les soutes, les substructions et les arrière-cours de ce qui semble nous établir si solidement dans ce que nous pensons être, et tout se met à vaciller; passons au cible de la méthode archéologique tout ce qui, naturellement, nous installe au centre et nous destine à l'universel, et tout devient douteux - replacée dans le temps long de l'aveu, des pratiques de l'aveu en Occident, rapportée à l'histoire du pouvoir psychiatrique, la psychanalyse n'est jamais que le dispositif singulier, dans le double sens du terme, dont se dote « une société singulièrement avouante ».

Dans le geste foucaldien, l'opération de découpe prime sur le «contenu » des espaces découpés. Ceux-ci - et c'est l'un des points auxquels s'arrime l'incrimination de " centrisme »- sont désignés sur un mode conventionnel, par le moyen d'un certain nombre de termes et expressions qui n'ont pas tous le même statut et qui trouvent leur place dans des champs théoriques différents. Ainsi, lorsque Foucault, dans Histoire de la folie, écrit «l'Europe ", il dessine une topographie qui est celle des pays auxquels s'est limitée sa recherche: la France, l'Angleterre, l'Allemagne, l'Italie, la Hollande pour l'essentiel - une topographie qui a des «bords » - l'Espagne, la Russie... Ce n'est pas un principe spirituel commun, un système politique, des "valeurs » communes qui font que cette «Europe" peut être nommée comme une entité, ce sont des pratiques communes, des dispositifs, un champ d'expérience partagée - c'est à la même époque 
qu'y apparaissent, ici et là, des maisons de force, des hôpitaux, des prisons dont les traits communs sont évidents.

Lorsque, dans le même livre, Foucault emploie des expressions comme «la culture européenne » ou « la culture occidentale », il opère à l'évidence une synthèse à un autre niveau. Il présuppose l'existence de plis, de "choix ", de bifurcations, de gestes... se manifestant à des niveaux différents de la pensée et des pratiques collectives et trouvant leur «champ d'action » ou leur terrain d'exercice dans des espaces dont le propre est d'être moins bien spécifié que dans le cas précédent - à l'évidence, l'« Occident », « la culture occidentale », l'« amour occidental » sont des entités plus flottantes qu'une entité Europe dont les contours sont vérifiables dans les notes du livre - elle coïncide très précisément avec le champ d'extension de l'enquête, les livres lus, les archives dépouillées, etc. On identifie bien dans ce saut d'une notion à l'autre quelque chose comme l'élément d'une décision: il y a non seulement "de l'Europe " identifiable dans un champ d'expérience, un champ pratique et stratégique, mais il y a aussi non seulement «de la» mais une, la culture européenne et, par extension ou globalisation, de l'Occident, un Occident... une notion dont l'existence, la consistance se vérifierait par celle des plis, des gestes, des bifurcations, des champs pratiques tels qu'ils s'analysent topologiquement: des représentations et des usages de la folie dans telle séquence, des formes des savoirs, un certain type de discours sur le sexe - toujours en rapport à des singularités, donc, dont la texture est culturelle, historique - en ce sens, oui, ce Foucault-là est bien "culturaliste " - mais à condition de considérer la culture comme un champ de dispersion et d'inclusion, plutôt que de réalisation de quelques valeurs cardinales...

11 Mais il existe aussi toutes sortes d'autres termes et expressions par lesquels Foucault opère ses découpes. Lorsqu'il met en avant l'indice du "moderne" ("sociétés modernes ", " pouvoirs modernes »...), il fait basculer subrepticement de la topologie objective à la topologie subjective, d'un «Ici et pas ailleurs " à un «nous et pas les autres » (ou d'autres). Il dessine l'espace du moderne/contemporain comme condition, notre condition. Ce n'est pas du tout selon une approche historiciste, chronologique, que se saisit le moderne, mais bien plutôt sous l'angle du "nous»-le seuil de modernité, écrit-il dans Les mots et les choses, c'est là où nous éprouvons, dans notre condition de contemporain, c'est-à-dire dans un rapport infiniment variable et intensifiable à nous-mêmes et à notre époque, que se situe le point de passage « de notre préhistoire (je souligne) à ce qui nous est contemporain" - là où nous éprouverons, disons, que Nietzsche est notre contemporain, encore et toujours - et Hegel plus tout à fait... La découpe vise ici à faire apparaître les conditions d'un « nous » (toujours situé, délimité, mais sur un mode qui n'est plus aussi distinctement "géographique ») dont Foucault a décrit la particularité, la singularité " historique » dans son texte/commentaire de la fameuse "Réponse » de Kant à la question "Qu'estce que les Lumières?».

12 Cette articulation entre le «Ici» et le «nous» est décisive dans l'analytique foucaldienne. Le passage du «moment» de la description topologique à celui de la prolifération des "nous ", "nos ", " notre » (etc.) signale une inflexion décisive du geste philosophique. Ce n'est pas pour rien que l'usage du «nous » va en s'intensifiant, de L'histoire de la folie à La volonté de savoir, un essai qui s'ouvre sur cette phrase: «Longtemps, nous aurions supporté, et nous subirions aujourd'hui encore, un régime 
victorien » et qui s'écrit tout entier sous le signe de ce pluriel (collectif) et des pronoms possessifs qui s'y rapportent.

Si les découpes topologiques demeurent constantes d'un ouvrage à l'autre (le «nous » de La volonté de savoir est un « nous » français, européen, occidental...), le geste change pour autant que le «nous » est un intensificateur du récit et de l'analyse qui implique le lecteur sur un autre mode que, disons, l'archéologie froide et savante qui entend son empire sur Les mots et les choses. Le jeu avec la singularité du topos se déploie dans La volonté de savoir sous la forme de la présentation de conditions d'appartenance auxquelles le lecteur (français, européen, occidental) ne saurait se soustraire. C'est sur ces conditions mêmes qu'il est convié à réfléchir, afin de s'en desceller dans la mesure du possible - «Il faudrait se demander aussi pourquoi nous nous culpabilisons si fort aujourd'hui d'en [le sexe] avoir fait autrefois un péché ?».

Ce «nous» découpe l'espace dans lequel les contemporains (occidentaux) de l'auteur sont convoqués à se saisir sur un mode réflexif et critique de ce qui constitue la trame d'une actualité qui les fait ce qu'ils sont, à faire de cette actualité leur problème, à la problématiser en tant qu'elle balise leur condition. C'est donc un «nous" d'interpellation qui «nous» convie à nous décentrer par rapport aux évidences fondatrices de notre commune constitution ("Nous autres, supposés victoriens...»), à déconstruire ou détricoter maille par maille le tissu de ce partage fallacieux des sensibilités dont est fait «ce que nous sommes " (sexuellement réprimés, avides de notre «libération »...). Il s'agit bien de cette sorte d'ascèse par laquelle nous sommes conviés à nous exercer à voir d'ailleurs ce qui s'impose à nous comme principe constitutif de notre existence, de notre champ d'expérience :

Et nous devons songer qu'un jour, peut-être, dans une autre économie des corps et des plaisirs, on ne comprendra plus bien comment les ruses de la sexualité, et du pouvoir qui en soutient le dispositif, sont parvenues à nous soumettre à cette austère monarchie du sexe, au point de nous vouer à la tâche indéfinie de forcer son secret et d'extorquer à cette ombre les aveux les plus vrais.

On en conviendra : cet appel adressé au sujet occidental à se dés-assujettir de ce qui constitue son champ d'évidence propre n'est pas là la pire des versions de l'occidentalisme, de l'eurocentrisme. Il est donc temps de s'interroger sur le paradoxe d'une attaque qui prend pour cible un des philosophes européens contemporains qui est allé le plus loin dans la destruction (démolition) des présupposés non questionnés des gestes philosophiques en Occident, alors même que prospèrent sous nos latitudes toutes sortes de philosophies de bon renom ou de bon rapport qui attestent la persistance insouciante ou agressive de ce type de posture de la philosophie, de cet immémorial occidentaliste/orientaliste de la philosophie européenne.

Lorsque, à l'occasion d'une conférence intitulée «Mondialisation, civilisations : quelles valeurs pour le $\mathrm{xxI}^{\mathrm{e}}$ siècle ? », le philosophe à succès André Comte-Sponville énonce le sophisme suivant: «Parce que s'il est vrai que tous les êtres humains sont égaux en droit et en dignité, alors une civilisation qui affirme cette égalité en droit et en dignité de tous les êtres humains, est supérieure [je souligne, $\mathrm{AB}$ ], du moins de ce point de vue, à une civilisation qui nie cette égalité ou qui la viole systématiquement", il s'inscrit lourdement, massivement, dans la perspective d'un "occidentalisme » idéologique dont le principe est l'existence manifeste d'une supériorité structurelle et constante de la « civilisation » occidentale sur tout autre. Lorsqu'il explicite son propos en affirmant que « [...] s'il faut choisir entre la civilisation qui est la nôtre, la civilisation occidentale ou judéo-chrétienne d'aujourd'hui, et la civilisation que prétend symboliser un Ben 
Laden, croyez bien que de mon point de vue le choix est vite fait. Et je n'ai aucune hésitation à dire que notre civilisation est supérieure à celle que prétend symboliser Ben Laden", les choses sont tout à fait claires et la "messe » occidentaliste dite passez-moi l'expression.

Mais aussi bien, le préjugé eurocentrique et occidentaliste se livre à cru chez un penseur de meilleure tenue que Comte-Sponville, Cornelius Castoriadis, déclinant, du ton de l'évidence : «Nous imitons des paradigmes, des modèles, et nous n'avons pas à imiter Athènes. Mais voilà : nous pouvons peut-être faire quelque chose de ces germes, alors que nous pouvons difficilement faire quelque chose de la politique des empereurs Tang, quels que soit par ailleurs son intérêt historique et sociologique, l'art remarquable qui a fleuri pendant cette période, etc.». Est-il besoin d'une longue investigation pour détecter que le "nous" autocentré et content de l'être sous la plume d'un auteur lui-même de provenance hellénique, le hasard faisant toujours bien les choses, présente un vif écart avec celui qui prolifère dans les textes de Foucault évoqués ci-dessus?

Dès lors, une question se pose avec insistance : de quel geste herméneutique, critique relève la démarche consistant à repérer la tache de sang intellectuelle de l'eurocentrisme et de l'occidentalisme («extrêmes») chez celui de nos auteurs contemporains qui, avec Deleuze, et d'un mouvement infiniment plus radical que ceux qui se repèrent chez des auteurs comme Derrida, Nancy, Agamben, Rancière ou Badiou, a lié le destin de son travail philosophique à une ontologie de nous-mêmes indissociable du dés-assujettissement, au descellement, à la quête, sinon d'un point d'extériorité, mais du moins d'une limite, d'un bord - là où, précisément, tombent tous les voiles de ce qui nous établit, nous destine et nous institue au « centre »?

La réponse à la question posée s'énonce assez simplement : ce geste, familier, c'est celui de l'herméneutique sans qualité, de la symptomatologie déchaînée qui prospère aujourd'hui, et dont le principe dévastateur est simple: tout fait symptôme, et plus celui-ci se dérobe, se présente comme subreptice, furtif, plutôt que manifeste et massif, plus il nous conduit droit au cœur du « refoulé » où gît le secret des choses. Et donc, plus se dérobe, chez des auteurs comme Deleuze et Foucault, le préjugé occidentaliste et eurocentrique, plus il se replie vers ces strates profondes où le philosophe ignore « d'où il parle » et où les mots piégés parlent à travers lui, irriguent et contaminent son discours à son corps défendant, et plus la chasse au symptôme sera excitante et sa détection aura de prix. Or, comme chacun sait, la règle première de ce jeu est que tout fait symptôme, l'intérêt marqué de Foucault pour les supplices d'Ancien Régime au temps de la monarchie absolue anglaise ou française plutôt que pour ceux pratiqués en Chine sous la dynastie Qing peut devenir la preuve accablante de la persistance du pli eurocentrique dans sa recherche. C'est un peu comme si, en anthropologie, le partage entre un parti « occidentaliste » et un autre qui ne le serait pas recouvrait la distinction entre les chercheurs qui persistent à s'intéresser aux tribus lointaines et ceux qui se consacrent à une ethnographie du RER...

Cette musique qui est comme le déchet des déchets de l'événement discursif associé au nom de Freud (relayé à l'occasion par ce "derridisme » tous usages et mondialisé qui prospère sous les latitudes des nouveaux mondes universitaires) nous est familière. C'est la même qui nous est servie lorsque nos protestations contre le dernier des massacres perpétrés par l'armée de l'État d'Israël à Gaza sont diagnostiquées par de 
savants docteurs pas occidentalistes pour un sou comme le symptôme d'un antisémitisme d'autant plus pernicieux qu'il ne s'énonce jamais.

21 Je remarque au passage que lorsque Foucault, à l'occasion de ses deux voyages au Japon, présente des conférences dans des universités, dialogue avec des universitaires locaux, est interviewé par des journalistes, ne met jamais en scène l'opposition Occident/Orient qui est, pour les disciples d'Edward Saïd, la marque de fabrique de l'occidentalisme et de son jumeau, l'orientalisme. Il dit, toujours aux mêmes conditions d'une géographie élémentaire et circonspecte, "Extrême-Orient ", " votre société », " le Japon », etc. Je vais m'attarder un peu sur un texte qui montre comment, dans ce genre de situation de communication interculturelle, il met en place un dispositif destiné à étouffer dans l'œuf la tentation occidentaliste.

Dans ce texte, donc, intitulé «La philosophie analytique de la politique ", une conférence prononcée au Japon en 1978, Foucault envisage sa propre position d'énonciateur philosophique ou, plus généralement, de publiciste, dans sa relativité même à d'autres situations, d'autres conditions, d'autres conditions d'énonciation. Évoquant la question des prisons, du système pénitentiaire auquel il a, au cours des années précédentes, consacré un livre et une partie importante de son action publique, il témoigne de la façon suivante de l'inclusion de l'enjeu du relatif (ou du décentrement) dans le champ de ses préoccupations et à la fois dans sa démarche intellectuelle et son engagement : ayant visité deux prisons dans la région de Fukoka, il s'est « aperçu que le problème de la pénalité, de la criminalité, de la prison se posait [au Japon, donc] dans des termes très différents dans votre société [il s'adresse à ses interlocuteurs japonais] et dans la nôtre ». Pour cette raison, il a renoncé à son projet initial de présenter une conférence sur « le problème particulier des prisons ». Ce qu'il en aurait à dire, en effet, demeurerait entièrement captif de l'environnement européen dans lequel il a été élaboré et risquerait, par conséquent, d'échouer face à l'épreuve de sa transposition dans ce monde « différent ».

Sa conférence, Foucault va donc la transformer en ce difficile exercice : l'examen de la «question du pouvoir », la présentation de quelques propositions et concepts ayant trait à une analytique de la politique, sous condition de soumission à l'épreuve de l'hétérogène, du différent - spécifiquement d'une différenciation/opposition entre «Occident» («Europe », " nos sociétés ", " chez nous» " pays occidentaux »...) et « Extrême-Orient » (" Japon », " sociétés d'Extrême-Orient »). Les motifs, les analyses, les concepts qu'il va présenter dans cet exposé sont donc, dans leur déploiement, constamment suspendus à cette réserve ou cette condition générale: c'est une « histoire » de l'Occident qui est ici « racontée » selon un mode ou dans un style qui est celui de la philosophie; si cette «histoire» peut avoir une valeur pour un public extrême-oriental, si celui-ci peut y repérer des paradigmes ou y trouver des stimulations, ces opérations ne se feront pas sur le mode de la transposition, mais plutôt sur celui de l'examen des écarts, des différences, voire des contrastes. Ce qu'il s'agit de pratiquer, ce ne sont pas des concepts ou des séquences analytiques "forts » en ce sens que s'imposerait tout naturellement leur universalité, mais bien un art de faire émerger des singularités, dans leur vocation à être mises en relation les unes avec les autres.

24 Ainsi, ayant évoqué ces " grandes maladies " du pouvoir qu'ont été, au $\mathrm{xx}^{\mathrm{e}}$ siècle et en Occident, le fascisme et le stalinisme, ayant montré comment, en Occident encore, la philosophie en était venue à lier son destin à celui de l'État, ayant parcouru à grandes 
enjambées l'histoire du pouvoir pastorat, dans sa relation notamment au christianisme, Foucault ébauche un programme de recherche comparatif : « [...] Il vaudrait la peine de comparer le pastorat, le pouvoir pastoral des sociétés chrétiennes avec ce qu'a pu être le rôle et les effets du confucianisme dans les sociétés d'Extrême-Orient ». Dessinant les linéaments d'une telle analyse, il ajoute :

Il faudrait faire la différence [je souligne] entre le pouvoir pastoral et le confucianisme : le pastorat est essentiellement religieux, le confucianisme ne l'est pas ; le pastorat vise essentiellement un objectif situé dans l'au-delà et n'intervient ici-bas qu'en fonction de cet au-delà, alors que le confucianisme joue pour l'essentiel un rôle terrestre; le confucianisme vise une stabilité générale du corps social par un ensemble de règles générales qui s'imposent à tous les individus ou à toutes les catégories d'individus, alors que le pastorat établit des relations d'obéissance individualisées entre le pasteur et son troupeau...

Peu importe ici le caractère plus ou moins précaire des termes de la comparaison que suggère ici Foucault entre pastorat chrétien et le confucianisme dans sa relation au gouvernement des vivants. Ce qui compte, c'est l'inclusion dans le champ de sa recherche de l'élément de la relativité, le travail à l'œuvre, fût-ce sur un mode implicite de notions comme celles de l'hétérogène, du différent, du discontinu, du singulier.

Pour Foucault, ce qui caractérise une société ou un système de pensée, c'est davantage ce qu'il rejette ou exclut que ce qu'il affirme et valorise. Son travail sur les sociétés européennes, sur les systèmes de pensée dans nos pays est fondé sur cette prémisse. Il découle de cette position que la recherche et l'identification des écarts significatifs entre la manière dont une société fait le partage entre ce qu'elle exclut et ce qu'elle valorise et la façon dont une autre met en œuvre cette opération est inscrite au cœur de sa réflexion. Discutant du statut de la folie dans la société française et dans la société japonaise avec M. Watanabe, un philosophe japonais, il dit: «Le fait que, dans votre pays, le fou soit le représentant du sacré et, dans le nôtre, le porteur de la vérité me paraît indiquer une différence significative [je souligne, $A B]$ entre la culture japonaise et la culture européenne ». On peut naturellement objecter que l'on est là dans un domaine de généralités forcément approximatives, comme lorsque, dans le texte cité plus haut, Foucault définit le stalinisme comme une maladie de l'État occidental, mais on ne peut certainement pas dire que c'est le préjugé occidentaliste qui conduit le raisonnement ici. Ce qui intéresse Foucault, c'est d'identifier des éléments de discontinuité, de redonner sa pleine dimension à l'hétérogène, de façon à ce que, du cœur même de nos identités " compactées " par les routines de pensées mais aussi par les dispositifs de gouvernement des vivants, nous redevenions sensibles à la présence de formes d'altérité radicale ou d'" espaces autres", radicalement autres, dans le domaine des pratiques culturelles, des systèmes de pensée, des modes d'organisation sociale, etc. Il est vrai que ce souci de l'hétérogène et du discontinu qui porte au delà de la reconnaissance de l'élément de la pluralité conduit inéluctablement Foucault à rendre compacts les éléments qu'il oppose, ou alors à mettre l'accent sur des objets porteurs de celle-ci - l'hôpital général, la prison pénitentiaire moderne, le panoptique, le discours du sexe réprimé, etc. On peut toujours objecter qu'à y regarder de plus près, "les choses sont toujours plus compliquées", comme on dit, que le ferment de l'hétérogène travaille toujours au cœur de l'homogène - ce que Foucault était toujours le premier à admettre - voir ses variations dans l'analyse du panoptique - mais à la condition expresse de ne pas placer une telle critique sous le signe d'un stigmate qui coupe court à toute objection ou contre-argumentation, tant il est, dans les conditions 
actuelles, propre à discréditer celui auquel il s'applique et irréfutable, de par son caractère infiniment variable et indéterminé.

Pour finir sur une note qui nous renvoie au plus extrême contemporain, je voudrais citer brièvement un article écrit par Foucault en 1979 et qui appartient à la série de ses " reportages d'idées » sur le soulèvement iranien et la chute du Chah, un texte intitulé "Une poudrière appelée Islam », un article qui témoigne, me semble-t-il, de l'extrême sensibilité de notre auteur à ce que l'on pourrait appeler l'absolument autre, autre de nous-mêmes, ou plutôt de ce que nous sommes supposés être, bref, au motif hétérotopique. Ayant relevé la rareté de l'événement consistant en le soulèvement d'un "peuple sans armes qui se dresse tout entier et renverse de ses mains un régime 'tout puissant' " sans se conformer d'aucune manière à "un modèle 'révolutionnaire' reconnu ", il ajoute cette réflexion dont chacun se sentira libre d'évaluer l'écho qu'elle peut rencontrer dans notre présent : «L'Islam - qui n'est pas simplement une religion, mais un mode de vie, une appartenance à une histoire et à une civilisation - risque de constituer une gigantesque poudrière, à l'échelle de centaines de millions d'hommes. Depuis hier, tout État musulman peut être révolutionné de l'intérieur, à partir de ses traditions séculaires ».

Ce qui caractérise et qualifie un geste philosophique qui tranche, c'est qu'il ouvre un espace de pensée nouveau inédit, qu'il est doté d'une force propulsive propre qui le destine à être relayé à l'infini par ceux qui le perçoivent et s'en saisissent. Dans un geste philosophique doté de cette puissance propre se trouvent inextricablement mêlés des concepts nouveaux qui le soutiennent, mais aussi, constamment, des mots de toutes sortes empruntés au vocabulaire courant, des mots valises, des mots puissants mais vagues, etc. mais des mots qui n'en sont pas moins nécessaires pour que le discours puisse s'articuler, de la même façon qu'il est bien difficile qu'un discours politique s'articule sans être soutenu par le mot "peuple »-signifiant vide par excellence, ou trop plein, ce qui revient au même. Chez Foucault, donc, ce n'est pas par l'effet d'une négligence ou d'une panne de la pensée que les concepts « créés » qui soutiennent son analytique $\mathrm{du}$ présent (biopouvoir, biopolitique, gouvernement des vivants, gouvernementalité, disciplines, dispositifs, mécanismes de sécurité...) côtoient des termes qui n'ont pas du tout le même statut, qui ne sont pas des concepts mais des éléments de descriptions de la découpe qui constitue la condition du travail philosophique.

\section{RÉSUMÉS}

Ce texte remet en cause une lecture de l'œuvre de Michel Foucault inspirée par les études postcoloniales, fondée sur la notion d'une perspective " eurocentrique ", « occidentaliste » inhérente à cette œuvre. Il tente de montrer comment le travail archéologique de Foucault procède d'opération de découpe et de topologisation des objets étudiés qui incluent l'élément du relatif et la dimension de l'historicité. Il présente toutes les conditions qui entourent l'énonciation d'un «nous » dans l'œuvre de Foucault. 
INDEX

Mots-clés : découpe, études post-coloniales, eurocentrisme, Europe, geste, Occident, occidentalisme

Personnes citées : Foucault (Michel)

\section{AUTEUR}

ALAIN BROSSAT

Professeur de philosophie, université Paris 8 Saint-Denis, abrossat@club-internet.fr 